\title{
Gray matter volume reduction with different disease duration in trigeminal neuralgia
}

\author{
Shanshan Shen ${ }^{1,2} \cdot$ Huifeng Zheng ${ }^{3} \cdot$ Jianwei Wang $^{4} \cdot$ Wenchang Guo ${ }^{5} \cdot$ Xiaowan Guo $^{1,2} \cdot$ Hong Ji $^{1,2}$. \\ Shuqian Zhang ${ }^{1,2} \cdot$ Yingmin Chen $^{1,2} \cdot$ Gaofeng Shi ${ }^{1}$ (]
}

Received: 30 May 2021 / Accepted: 30 July 2021 / Published online: 28 August 2021

(c) The Author(s), under exclusive licence to Springer-Verlag GmbH Germany, part of Springer Nature 2021, corrected publication 2021

\begin{abstract}
Purpose Structural magnetic resonance imaging is widely used to explore brain gray and white matter structure in trigeminal neuralgia (TN) but has yielded conflicting findings. This study investigated the relationship between disease duration as a clinical feature of TN and changes in brain structure.

Methods We divided $49 \mathrm{TN}$ patients into three groups (TN1-TN3) based on disease duration (TN1 = 1.1 $\pm 0.7(0-2)$ years, $\mathrm{TN} 2=4.8 \pm 1.5(3-7)$ years, $\mathrm{TN} 3=15.1 \pm 5.5(10-30)$ years). We used voxel-based morphometry (VBM) to compare the gray matter volume (GMV) across groups and between TN patients and 18 matched healthy control subjects.

Results The TN1 group showed reduced GMV of pain-related regions in the cerebellum; the TN2 group showed reduced GMV in the thalamus and the motor/sensory cortex; and the TN3 group showed reduced GMV in the emotional and reward circuits compared with healthy controls. Similar brain regions, including bilateral hippocampi, caudate, left insular cortex, and medial superior frontal cortex, were affected in TN2 and TN3 compared with TN1.

Conclusion Disease duration can explain differences in structural alterations-especially in pain-related brain regions-in TN. These results highlight the advanced structural neuroimaging method that are valuable tools to assess the trigeminal system in TN and may further our current understanding of TN pathology.
\end{abstract}

Keywords Trigeminal neuralgia $\cdot$ Pain $\cdot$ VBM $\cdot$ Structure $\cdot$ Imaging

\section{Highlights}

1. Trigeminal neuralgia $(\mathrm{TN})$ is a severe neuropathic pain disorder; the pathogenesis is not fully understood.

2. Structural MRI has been used to exhibit the gray matter changes in $\mathrm{TN}$, but results were different in previous studies.

3. We show that the disease duration is one of the causes for the gray matter differences and further exhibits the order of brain regions in pain processing in different disease durations.

\section{Gaofeng Shi}

447546952@qq.com

1 Department of Radiology, Fourth Affiliated Hospital of Hebei Medical University, Shijiazhuang, Hebei, China

2 Department of Radiology, Hebei General Hospital, Shijiazhuang, Hebei, China

3 Department of Radiology, Shexian Hospital, Handan, Hebei, China

4 Department of Radiology, Xianghe Hospital, Langfang, Hebei, China

5 Department of Neurosurgery, Hebei General Hospital, Shijiazhuang, Hebei, China

$\begin{array}{ll}\text { Abbreviations } \\ \text { ACC } & \text { Anterior cingulate cortex } \\ \text { BOLD } & \text { Blood-oxygen level dependent } \\ \text { CC } & \text { Cingulate cortex } \\ \text { CSF } & \text { Cerebrospinal fluid } \\ \text { DARTEL } & \text { Diffeomorphic Anatomical Registration } \\ & \text { Through Exponentiated Lie Algebra } \\ \text { GM } & \text { Fray matter } \\ \text { GMV } & \text { Gray matter volume } \\ \text { HCs } & \text { Healthy control subjects } \\ \text { MRI } & \text { Magnetic resonance imaging } \\ \text { OFC } & \text { Orbitofrontal cortex } \\ \text { PFC } & \text { Prefrontal cortex } \\ \text { S1 } & \text { Primary somatosensory cortex } \\ \text { TA } & \text { Acquisition time } \\ \text { TE } & \text { Echo time } \\ \text { TIV } & \text { Total intracranial volume } \\ \text { TN } & \text { Trigeminal neuralgia } \\ \text { TR } & \text { Repetition time } \\ \text { VAS } & \text { Visual analog scale } \\ \end{array}$


VBM Voxel-based morphometry

WM White matter

\section{Introduction}

Trigeminal neuralgia (TN) is a severe neuropathic pain disorder characterized by intense electric shock-like pain in one or more trigeminal distributions [1]. As the disorder progresses, pain becomes more frequent and sustained, increasing the risks of anxiety and depression and greatly diminishing patients' quality of life [2,3]. The pathogenesis of TN is not fully understood, and it is not known whether it originates in the central or peripheral nervous system.

Neuroimaging techniques such as structural and functional magnetic resonance imaging (fMRI) are effective tools for exploring the mechanisms underlying the development and progression of TN. Diffusion tensor imaging (DTI) has been used to evaluate the plasticity of the brain white matter (WM), axial kurtosis, and axial diffusivity in the sensory and cognitive aspects of pain [4, 5]. Voxel-based morphometry (VBM) has been used to measure gray matter volume (GMV) and cortical thickness in chronic pain such as changes in GM in the thalamus, hippocampus, cerebellum, and anterior cingulate cortex (ACC) associated with pain processing and emotional perception [6-8]. Functional connectivity and the amplitude of low-frequency fluctuations (ALFF) can reflect neuronal activity and are correlated with hemodynamic fluctuation [9]. The alteration of brain structure and function are associated with chronic pain. However, the relationship between brain reorganization and chronic pain is not fully understood.

Human brain imaging studies have been carried out with the aim of identifying markers for pain processing, but the reported findings have varied [10-12]. For example, various studies have reported that GMV in the thalamus was decreased [2, 6, 13], increased [14, 15], or showed no changes $[3,16,17]$ in patients with chronic pain, such as TN. These conflicting results may be partially due to the focus on different brain regions such as the thalamus, hippocampus, insula, ACC, prefrontal cortices (PFC), basal ganglia, and amygdala. Additionally, drugs used for treatment, the surgical history, disease duration, and the severity and pattern of pain are major reasons for the discrepancies reported by brain imaging studies $[10,18]$. Differences in pain sensitivity and abnormal regions have also been demonstrated between men and women $[19,20]$. Thus, more longitudinal studies integrating different imaging techniques are needed to clarify the neural basis of pain in $\mathrm{TN}$.

In addition, to explain the differences in gray matter between previous studies, we identified the brain structures involved in pain and pain-related regions. Alterations in GMV over time have been reported [8]. These changes are not in a specific region but involve the whole brain and reflect the variable susceptibility of different cortical structures. While decreases in GMV are likely to be the consequence and not the cause of pain [21], evaluating the dynamics of GMV decreases such as that in TN could provide etiological insight and guide management strategies.

In the present study, we evaluated GMV in patients with $\mathrm{TN}$ grouped according to disease duration and healthy control subjects (HCs) using structural MRI to clear the bias reported in the literature and identify changes in neural structures at different stages of TN.

\section{Patients and methods}

\section{Study design}

This single-center, prospective, cross-sectional study enrolled 49 patients diagnosed with idiopathic TN according to the international Classification of Headache Disorders, 3rd edition (ICHD-3) [22] (30 females; mean age: $58.4 \pm 11.5$ years) who were referred to neurosurgery department at Hebei General Hospital between October 2019 and June 2020, as well as 18 HCs (11 females; mean age: $59.8 \pm 8.0$ ) matched in terms of age, sex, and education level. All participants were right-handed. Patients with a history of multiple sclerosis or other lesions that could cause TN (e.g., brain tumor), cerebral hemorrhage, cerebral infarction, brain trauma, epilepsy, and other neurological or psychiatric disorders were excluded. All patients never received any form of surgery.

\section{Clinical characteristics and outcome assessment}

The 49 patients were divided into the following three groups based on mean disease duration: TN1 $(n=16)$, $1.1 \pm 0.7$ years; TN2 $(n=17), 4.8 \pm 1.5$ years; and TN3 $(n=16), 15.1 \pm 5.5$ years. The following demographic and clinical data were collected: sex, age, duration of TN, visual analog scale (VAS) score, and medications. The duration of TN was defined as the amount of time from the initial TN diagnosis to the preoperative brain MRI examination. Subjects were asked to rate the extent of their neuralgia on a visual analog scale (VAS) with scores ranging from 0 to 10 $(0=$ no pain and $10=$ maximum imaginable pain $)$.

\section{MRI data acquisition}

MRI scanning was performed by an imaging technician with 15 years of experience using a 3.0 T MRI scanner (Discovery 750w; GE Healthcare, Cleveland, OH, USA) with a standard 8-channel head coil. High-resolution wholebrain T1-weighted images were acquired using a 3D-T1 BRAVO sequence with the following parameters: matrix 
size $=256 \times 256$, field of view $=25.6 \mathrm{~cm}$, repetition time $(\mathrm{TR})=8.5 \mathrm{~ms}$, echo time $(\mathrm{TE})=3.2 \mathrm{~ms}$, flip angle $=13^{\circ}$, slice thickness $=1.0 \mathrm{~mm}$, and acquisition time $(\mathrm{TA})=3 \mathrm{~min}$ $15 \mathrm{~s}$. Subjects were instructed to close their eyes and not think about anything in particular and not to fall asleep. None of the patients reported falling asleep during the scan.

\section{MRI data analysis}

VBM analysis was performed with Statistical Parametric Mapping (SPM12 http://www.fil.ion.ucl.ac.uk/spm)-which including the VBM toolbox (http://dbm.beuro.uni-jena.de/ $\mathrm{vbm} /$ ) and Diffeomorphic Anatomical Registration Through Exponentiated Lie Algebra (DARTEL) algorithm using Matlab (The MathWorks, Natick, MA, USA). Preprocessed images were automatically segmented into GM, WM, and cerebrospinal fluid (CSF), spatially normalized with diffeomorphic anatomical registration through DARTEL to the subject-specific template and then smoothed with an isotropic Gaussian kernel of $8 \mathrm{~mm}$ full-width at half-maximum. Total intracranial volume (TIV), total GMV, WM volume, and CSF were calculated and averaged for each group.

\section{Statistical analysis}

Differences in age, sex, education level, and pain score between groups were evaluated with the Wilcoxon signedrank test. Differences in voxel-wise GMV images were analyzed with TIV, age, and sex as covariates with a two-sample $t$-test and by analysis of covariance using the Data Processing and Analysis for (resting state) Brain Imaging (DPABI) toolbox [23]. Significant clusters were identified using the threshold-free cluster enhancement (TFCE) method [24]. The threshold of corrected $P<0.005$ was applied, and correction for multiple comparisons at the cluster level was performed. All reported cluster coordinates were expressed in Montreal Neurological Institute (MNI) standard space.

\section{Results}

\section{Patient demographics}

Patients' demographic information is shown in Table 1. The majority of patients were taking medications for TN at the time of MRI examination if necessary; the most common drugs were carbamazepine and gabapentin. There were no significant differences among TN1, TN2, and TN3 and HCs groups in age or sex ratio $(P>0.05)$, and there were no significant differences in pain score among the three TN groups. There were six (37.5\%) left sides in the TN1 group, three (21.4\%) left sides in the TN2 group, and eight (50\%) left sides in the TN3 group.

\section{Alterations in GMV in TN}

GMV in the cortex differed significantly between TN patients and HCs $(P<0.005$; Table 2$)$. All of the abnormal regions are shown in Fig. 1. Only the cerebellum exhibited a lower GMV in patients (TN1 group) compared with HCs. The left thalamus, medial superior frontal cortex (MFC), bilateral postcentral, and orbitofrontal cortex (OFC) exhibited reduced GMV in patients (TN2 group) compared with HCs. Right anterior cingulate cortex, left hippocampus, bilateral insular cortex, and OFC exhibited reduced GMV in patients (TN3 group) compared with HCs. The distribution of the main regions is shown in Fig. 2. GMV in the cortex had similar abnormal regions between TN2 and TN3 compared with TN1 $(P<0.005$; Table 3$)$. GMV was reduced in bilateral hippocampi, caudate, left insular cortex, and MFC in the TN2 and TN3 group compared with the TN1 group (Fig. 3). All of the overlapping regions are shown in Fig. 4.

There were no significant differences in GMV between TN2 and TN3 groups, and no brain regions exhibited increased GMV at a threshold of uncorrected $P<0.005$.
Table 1 Demographics and clinical data of the participants

\begin{tabular}{lllll}
\hline & TN1 & TN2 & TN3 & HCs \\
\hline Number of subjects & 16 & 17 & 16 & 18 \\
Age (y) & $59.1 \pm 5.2$ & $60.5 \pm 10.0$ & $63.6 \pm 6.8$ & $59.8 \pm 8.0$ \\
Sex (F/M) & $10 / 6$ & $11 / 6$ & $9 / 7$ & $11 / 7$ \\
Disease duration, (y) & $1.1 \pm 0.7(0-2)$ & $4.8 \pm 1.5(3-7)$ & $15.1 \pm 5.5(10-30)$ & n.a \\
Sides (left/right) ${ }^{l}$ & $6 / 10$ & $3 / 14$ & $8 / 8$ & n.a \\
Pain score & $8.7 \pm 0.7$ & $8.6 \pm 0.9$ & $8.8 \pm 1.0$ & n.a \\
Medication & CBZ/GBP/CBZ\&GBP & CBZ/GBP/CBZ\&GBP & CBZ/GBP/CBZ\&GBP & n.a \\
\hline
\end{tabular}

$T N$ trigeminal neuralgia, $H C$ healthy controls, $C B Z$ carbamazepine, $G B P$ gabapentin, n.a. not applicable.

${ }^{l}$ Significant difference between-group revealed by chi-square test $(P<0.05)$. 
Table 2 Regions of reduced gray matter volume in TN1, TN2, and TN3 patients with trigeminal neuralgia compared to healthy controls $(P<0.005)$

\begin{tabular}{|c|c|c|c|c|c|c|}
\hline \multirow[t]{2}{*}{ Anatomical region } & \multicolumn{2}{|l|}{ Group one } & \multicolumn{2}{|l|}{ Group two } & \multicolumn{2}{|l|}{ Group three } \\
\hline & Peak coordinates $\mathrm{x}, \mathrm{y}, \mathrm{z}$ & T score & Peak coordinates $\mathrm{x}, \mathrm{y}, \mathrm{z}$ & $\mathrm{T}$ score & Peak coordinates $\mathrm{x}, \mathrm{y}, \mathrm{z}$ & T score \\
\hline Right cerebellum & $36,-69,-21$ & -5.3685 & & & & \\
\hline Left thalamus & & & $-9,-30,6$ & -7.7343 & & \\
\hline Left frontal_Tri & & & $-54,21,18$ & -4.5711 & & \\
\hline Left occipital & & & $-27,-69,30$ & -4.1925 & & \\
\hline Left postcentral & & & $-63,-9,24$ & -4.518 & & \\
\hline Right postcentral & & & $39,-30,39$ & -3.6242 & & \\
\hline Right MCC & & & $12,-24,39$ & -4.8762 & & \\
\hline Right precentral & & & $60,9,18$ & -6.4621 & & \\
\hline Left MFC & & & $-6,54,36$ & -5.4331 & & \\
\hline Right MFC & & & & & $9,57,30$ & -5.1205 \\
\hline Left OFC & & & $-33,30,-21$ & -5.367 & $-30,27,-21$ & -3.6248 \\
\hline Right OFC & & & $45,39,-18$ & -7.9674 & $45,39,-18$ & -5.5334 \\
\hline Left hippocampus & & & & & $-30,-30,-9$ & -8.0986 \\
\hline Left insula & & & & & $-33,-21,9$ & -7.3716 \\
\hline Right insula & & & & & $36,-15,6$ & -5.6427 \\
\hline Right ACC & & & & & $12,42,6$ & -4.7514 \\
\hline
\end{tabular}

MFC Frontal_Sup_Medial, OFC Frontal_Inf_Orb, ACC Cingulum_Ant, $M C C$ Cingulum_Mid.

\section{Discussion}

To the best of our knowledge, this is the first study to investigate the relationship between the duration of TN and changes in brain structure over time. There were three main findings: (1) TN patients were classified into three groups based on disease duration. As the pain severity and sex ratio of patients were similar to those reported in other studies, our results appeared to be reliable, suggesting that previously observed variations among $\mathrm{TN}$ patients can be explained by changes in brain structure occurring during TN progression. GMV decreased between TN1 $(<2$ years) and TN2 (3-7 years) and between TN1 and TN3 ( $>10$ years), but the difference between TN2 and TN3 was non-significant. (2) In the between-group analysis, the TN2 group showed engagement of the thalamicsomatosensory circuit compared with $\mathrm{HCs}$, as evidenced by patients' restricted movements of the trigeminal branches. Moreover, over time, the TN3 group was more reliant on the salience region, which involves emotion and reward circuits. Some overlapping brain regions were affected in both the TN2 and TN3 groups, including bilateral hippocampi, caudate, left insular cortex, and MFC. (3) The cerebellum, thalamus, and ACC are important in pain processing. GMV was reduced in these regions in $\mathrm{TN}$ patients compared with HCs. These findings demonstrated that brain reorganization in response to pain follows a bottom-up process of stimulus filtering.

\section{Reduced GMV in the cerebellum in TN1}

In the early stage of TN (disease duration of 1 year), GM was reduced only in lobule VI of the cerebellum. This is consistent with the findings of a meta-analysis [10] that included three studies reporting decreased GMV in the cerebellum. In two of these studies, the average reported illness duration of TN was $8.3 \pm 6.7$ years [13] and $5.8 \pm 6.3$ years (right side TN)/5.2 \pm 4.9 years (left side TN) [6]; the median duration was less than 2 years, indicating that many patients were in the early stage of disease. In the third study [25], the shortest TN duration was 2 years, but the median duration was 8 years; moreover, the average age of patients was 45.9 years (the youngest that has been reported), and there were fewer females than males. These factors may have caused the observed inconsistencies observed across studies [19]. The posterior cerebellum (e.g., lobules VI and VIIb) is involved in motor control and pain processing and directs pain-related action [26]. The cerebellum was shown to be activated in response to noxious heat and unpleasant images [27], demonstrating that it is sensitive to aversive stimuli, as well as responding to acute and chronic pain [28, 29] as a key component of the endogenous analgesia system. We found that the decrease in GMV in the cerebellum was not significant in the TN2 and TN3 groups compared with HCs, suggesting that other brain regions became more relevant with disease progression. In fact, it was shown that painassociated decreases in GMV were reversed after pain relief, for example, in the thalamus arthroplasty [30] and insular 


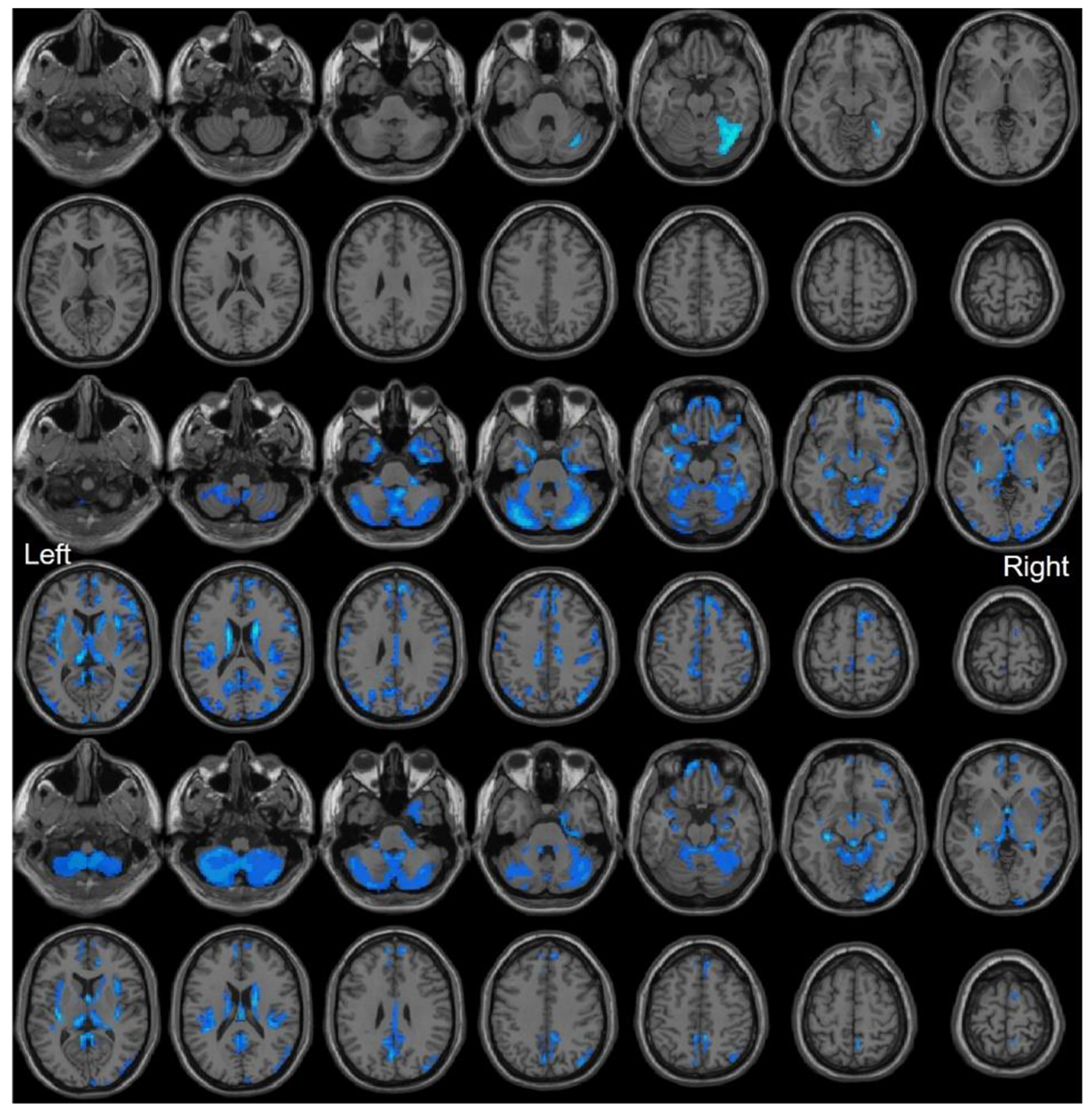

Fig. 1 GMV in TN patients compared to HC subjects evaluated by MRI. Images show reduced GMV in TN1 (top 2 rows), TN2 (middle 2 rows), and TN3 (bottom 2 rows) compared to HCs

cortex after surgery for the treatment of TN [31]. Thus, the cerebellum may not be involved in the processing of persistent pain, and its gray matter may reverse.

\section{GM changes in TN2}

The average duration of disease in TN2 was 5 years. In this group, GMV was decreased in the thalamus, OFC, visual cortex, middle CC, the primary somatosensory cortex (S1), and primary motor cortex (M1), in accord with previous studies $[2,6,13,15,16]$. Previous studies have reported that the GMV is reduced in the thalamus of patients with TN $[2,6,13]$ with an average duration of disease was of $5-8$ years, which is similar to the duration in the TN2 group. However, there was no decrease in GMV in the thalamus in $\mathrm{TN}$ patients with an average disease duration of 14 years 
Fig. 2 Comparisons of altered brain regions between $\mathrm{TN}$ groups and HCs. The blue triangle, yellow circle, and red diamond represent regions with reduced GMV in TN1, TN2, and TN3, respectively, compared to HCs

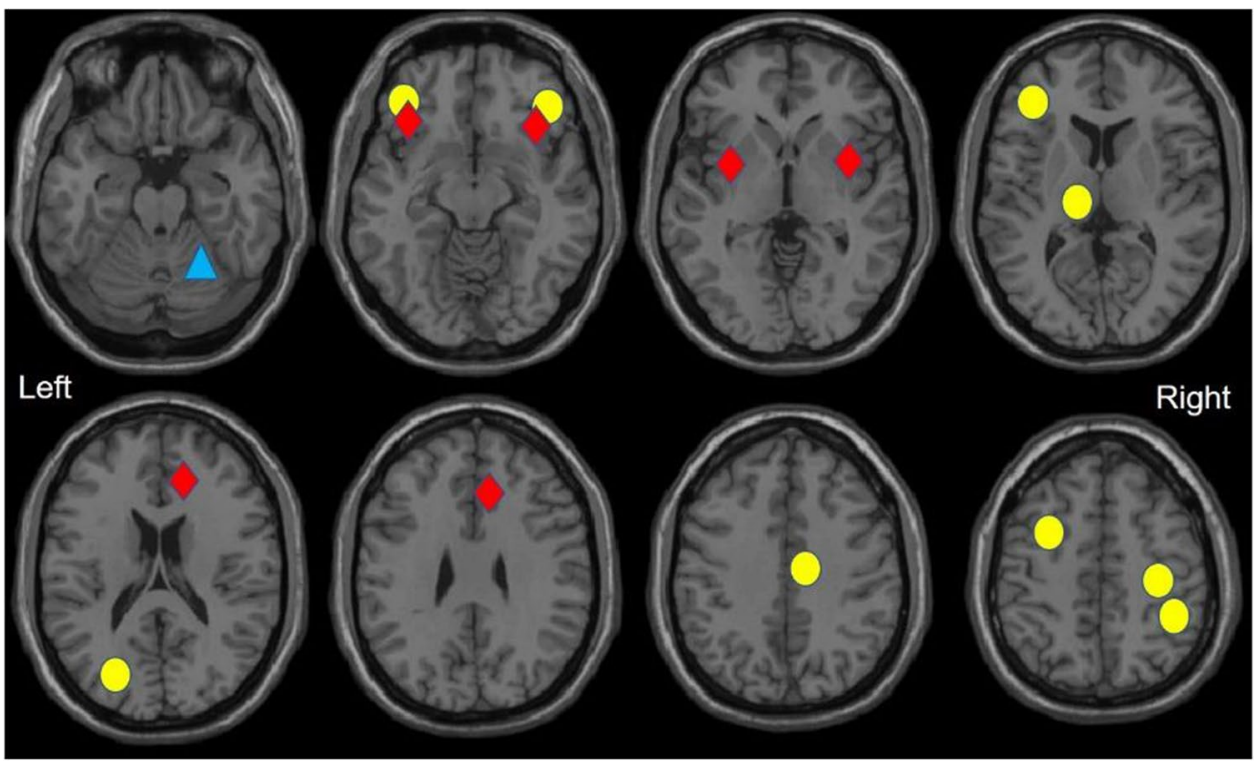

\begin{tabular}{|c|c|c|c|c|}
\hline \multirow[t]{2}{*}{ Anatomical region } & \multicolumn{2}{|l|}{ Group two } & \multicolumn{2}{|l|}{ Group three } \\
\hline & Peak coordinates $\mathrm{x}, \mathrm{y}, \mathrm{z}$ & T score & Peak coordinates $\mathrm{x}, \mathrm{y}, \mathrm{z}$ & $T$ score \\
\hline Left fusiform & $-30,-15,-33$ & -6.1442 & & \\
\hline Right fusiform & $33,-24,-27$ & -4.2576 & & \\
\hline Left OFC & & & $-45,36,-18$ & -6.3187 \\
\hline Right OFC & $24,24,-21$ & -3.7303 & & \\
\hline Left insula & $-30,15,9$ & -6.3422 & $-36,9,-9$ & -5.67 \\
\hline Right insula & & & $36,12,-12$ & -5.1253 \\
\hline Frontal_Inf_Tri_R & $51,39,0$ & -7.7918 & & \\
\hline Left hippocampus & $-33,-27,-12$ & -4.4969 & $-30,-30,-9$ & -8.6341 \\
\hline Right hippocampus & $33,-21,-15$ & -5.2303 & $33,-24,-12$ & -5.4367 \\
\hline Left caudate & $-15,0,18$ & -6.2381 & $-15,-9,21$ & -4.4499 \\
\hline Right caudate & $18,6,18$ & -6.4356 & $18,-12,21$ & -6.4314 \\
\hline Left MFC & $-9,66,6$ & -4.5826 & $-42,39,21$ & -4.6963 \\
\hline Right MFC & $-6,33,54$ & -4.1346 & & \\
\hline Right supp_Motor_Area & $9,15,63$ & -4.2373 & & \\
\hline Right MCC & $12,-24,39$ & -4.7621 & & \\
\hline Left calcarine & & & $-9,-45,3$ & -4.6657 \\
\hline
\end{tabular}

OFC Frontal_Inf_Orb, $M F C$ Frontal_Sup_Medial, $M C C$ Cingulum_Mid.
Table 3 Regions of reduced gray matter volume in TN2 and TN3 patients with trigeminal neuralgia compared to $\mathrm{TN} 1$ $(P<0.005)$
[16] (classified as the TN3 group in our study), consistent with our results. However, two studies reported that the GMV in the thalamus was increased in TN [14, 15]. It is likely that differences in data analysis methods, sex ratio, and symptom severity are responsible for these contradictory findings. The thalamus plays an important role in pain processing in TN [6]. We observed that GMV was reduced in the cerebellum but not in the thalamus in the TN1 group, whereas thalamic GMV was decreased in TN2 but not TN3. Thus, these two brain regions may be sequentially activated in the transmission of pain information, with the cerebellum initially responding to short-term pain followed by the thalamus responding to long-term pain. One neural area is activated, while adjacent areas are inhibited [32].

The thalamic-somatosensory circuity contributes to different aspects of pain perception [32]. External nociceptive stimuli induce the transmission of chemical substances and action potentials to the thalamus via $\mathrm{A}_{\delta}$ and $\mathrm{C}$ fibers [32], which project this information to the neocortex generally. A previous $f \mathrm{MRI}$ study demonstrated that, in patients with back 


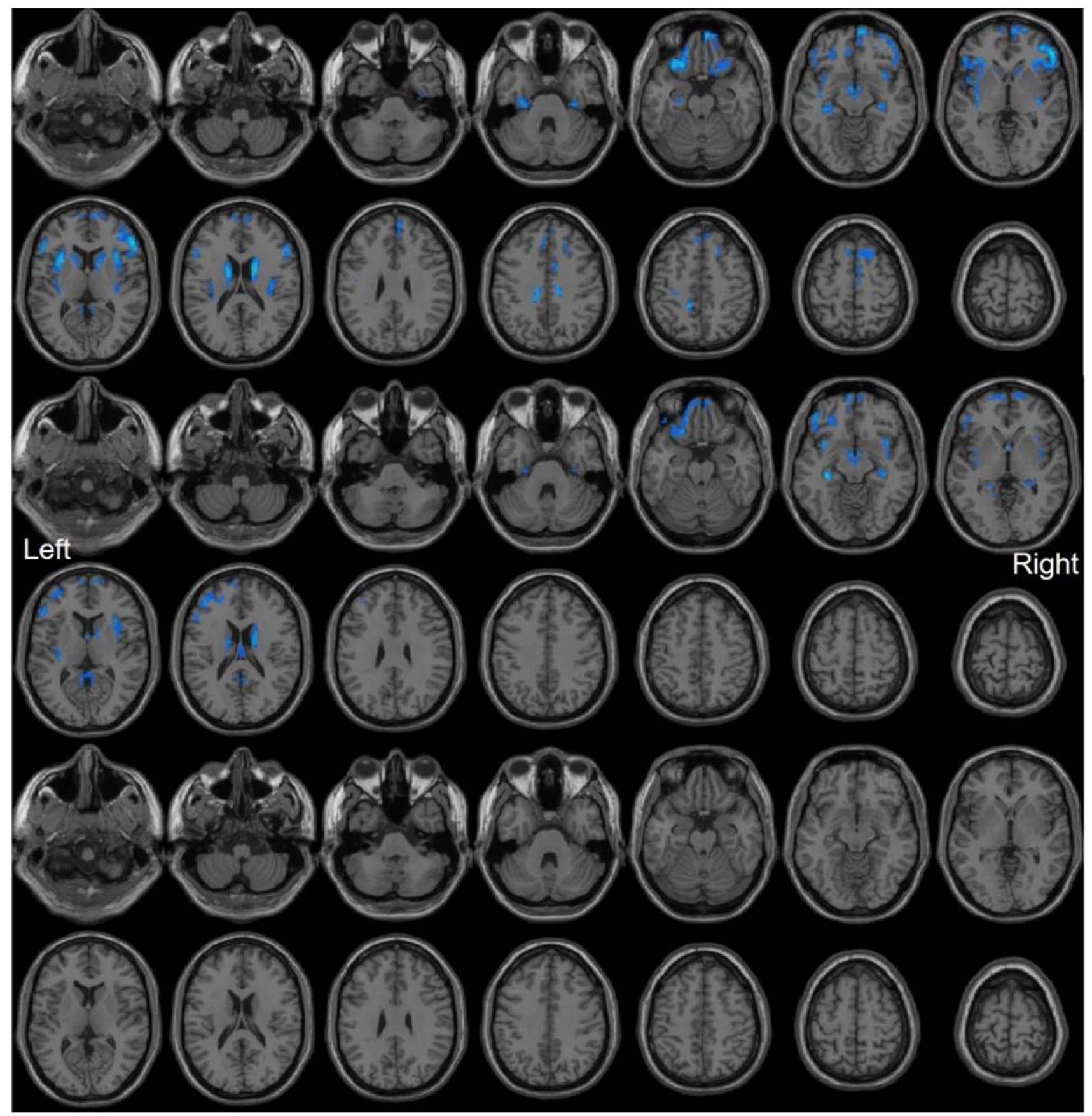

Fig. 3 Comparison of GMV between TN patient groups with different disease durations. Images show reduced GMV in TN2 vs TN1 (top 2 rows) and in TN3 vs TN1 (middle 2 rows). There was no difference in GMV between TN2 and TN3 (bottom 2 rows)

pain, the transition from the acute to the chronic stage was associated with a shift in brain activity from classical painrelated area to circuit more strongly related to the affective components of pain [33, 34]. Thalamic-somatosensory dysfunction was shown to reduce S1 activation [35]. Decreased neuronal activity is linked to reduce GMV $[32,36]$. GMV was shown to be lower in the sensorimotor areas of patients with persistent pain [37].

\section{GM changes in TN3}

In the persistent pain stage, the average duration of $\mathrm{TN}$ was 15 years; bilateral OFC, bilateral insula, left hippocampus, right ACC, and right medial PFC showed lower GMV, as reported in earlier studies $[13,16,38]$. The average disease duration in these previous studies ranged from 7.1 to 8.5 years, which is shorter than the disease duration for 


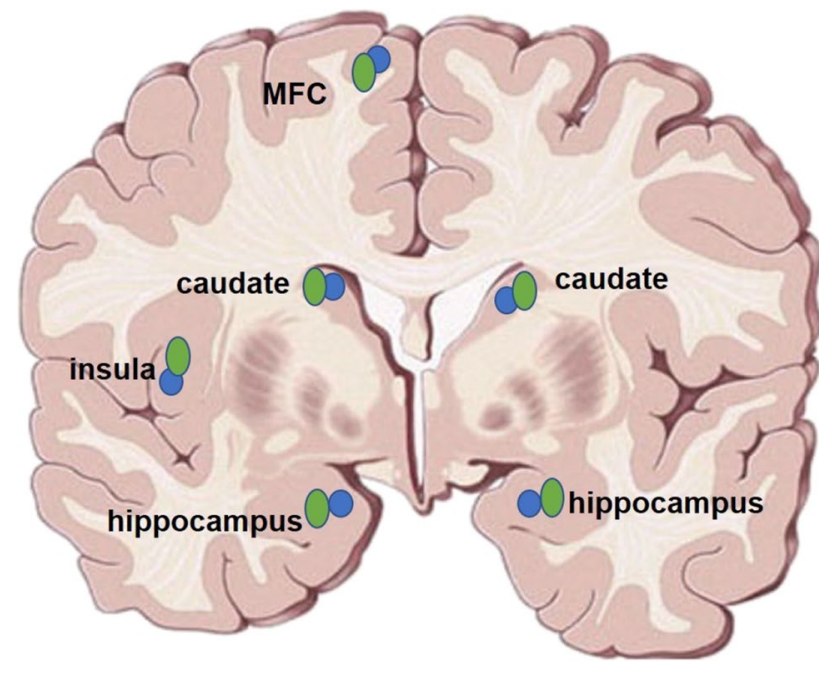

Fig. 4 Distribution of similar brain reions in intra-group comparisons (between TN1 and TN2, between TN1 and TN3). Blue and green represent comparisons between $\mathrm{TN} 1$ and $\mathrm{TN} 2$ and between $\mathrm{TN} 1$ and TN3, respectively

the TN3 patients in the current study. It is possible that the alterations observed in these previous studies would persist for 15 years or more.

Facial pain involves broad brain regions including the cingulate cortex (CC). The ACC receives input from the thalamus and projects to the entorhinal cortex [39]. Just as activation of the thalamus follows that of the cerebellum in the middle stage of pain processing, the ACC is activated after the thalamus during chronic pain. The role of the ACC in specific tasks is not fully understood [40]. Our results suggest that it is involved in the response to persistent pain. The ACC and insula form the salience region [41]; the anterior insula is thought to play a significant role in modulating emotions and stress [42]. The ACC, which is activated by noxious or painful stimuli [43], processes pain by interfering with cortical activity. The integrity of the salience region has been linked to better self-perceived physical health and quality of life as well as pain perception and discomfort [44]. The decreases in GMV in both the insula and right ACC observed in TN patients in the current study suggest impairment of the salience region.

The OFC is involved in emotion and reward processing [45]. The TN2 and TN3 groups showed decreased GMV in the OFC compared to HCs and the TN1 group, although there was no difference between them. Many patients with pain are unable to maintain a normal emotional state and are prone to depression [17]. Our results suggest that patients with a long duration of TN maintain stability in emotional control and regulation.

\section{GM alterations in different terms of chronic pain}

There was no significant difference in GMV between TN2 and TN3, but there were significant differences between TN1 and TN2 and betweenTN1 and TN3. Patients who had TN for less than 2 years made up less than half of the total sample size, and there appeared to be no relationship between GM and duration, vice versa. In the current study, the distributions of abnormal brain regions associated with the disease duration of the sample were in line with the previous literature [3, 6, 13, 14, 17, 25]. These findings may have been caused by adaptive mechanisms induced after the early onset of chronic pain stabilizing any neurodegeneration that occurred in the later stage of the disease.

In general, the cerebellum exhibited reduced gray matter in the TN1 group in the region near the trigeminal nerve. In addition, the thalamus took the relay baton (process pain) from the cerebellum, and the ACC maintained pain perception in the TN3 group rather than the cerebellum and thalamus. In previous studies, the thalamus has been reported to play a key role in relaying ascending inputs from the spinal cord to cortical areas, including the ACC $[32,36]$. Knudsen [46] proposed four basic procedures regarding the mechanism of attention, including automatic bottom-up filtering for salient stimuli. The current results suggest that the brain exhibited the same features of filtering pain, from the cerebellum to the thalamus and finally to the ACC.

The TN2 and TN3 groups showed reduced GMV in bilateral hippocampi, insula, and caudate compared with TN1. In a previous study demonstrating a correlation between GMV and disease duration, neuronal loss was suggested to be a consequence rather than the cause of pain [47]. The lack of differences between TN2 and TN3 could potentially have occurred because, in persistent pain, the rates of synapse elimination and formation are equal $[32,36]$, thereby halting the loss of neurons. How these changes affect neuronal function in other brain regions remains to be determined [48].

The caudate and insular cortex, as well as somatomotor and dorsal attention-related brain regions, are associated with persistent pain [49]. In the current study, the TN2 group showed reduced GMV in the caudate and insula cortex and somatomotor area, while the TN3 group only showed a reduction in the caudate and insula cortex. Structure alteration in the left caudate and insula cortex was shown to predict the evolution of pain intensity in chronic pain [50] within 3 months [51]. It was reported that brain activity patterns associated with acute and subacute pain did not overlap with those of chronic back pain [33]. In the current study, some patients had been affected by TN for 15 years, and there was overlapping activity in different 
brain areas throughout the long-term disease course, as previously reported [33].

Subcortical structures including the hippocampus and basal ganglia have been implicated in chronic pain [52]. The hippocampus is a key region for memory and learning, which are important aspects of chronic pain and depression [53] and relevant to their co-occurrence $[53,54]$. TN patients have been reported to exhibit higher depression and anxiety scores than HCs [17]. Importantly, more brain regions showed reduced GMV in TN2 compared with TN1 (bilateral hippocampi, insula, caudate, and temporal cortex) than in TN2 compared with HCs. We speculated that GMV was greater in the TN1 group compared with HCs because of increased neuronal proliferation induced by hyperactivation. This could explain the increased GMV observed in some previous studies of TN $[14,15]$. Our finding that the left hippocampus showed reduced GMV in TN3 compared with HCs while GMV in bilateral hippocampi was lower in TN3 compared with TN1 suggests that the left hippocampus was affected sooner by persistent pain than the right hippocampus.

The bilateral insula showed reduced GMV in TN3 but not TN2 compared with HCs, suggesting that there was a continuous loss of neurons in the insula. Similar to the hippocampus, the left insula was affected to a greater extent than the right insula in the TN2 and TN3 groups. Finally, the GMV in the PFC reduced in the TN2 group compared with the TN3 group suggests that ongoing pain-induced changes in synaptic activation or function, which has been linked to diminished cognitive abilities and emotional control[55], although patients with chronic TN may experience partial recovery as a result of adaptation.

\section{Limitations}

There were several limitations in the current study. First, in the TN1 group, five patients had TN for only 3 months and one patient had been diagnosed just 1 week before enrollment. Thus, their pain symptoms were acute [56] and involved brain regions that are distinct from those associated with chronic pain [33]. As a result, there may have been a bias toward positive results in the TN1 group. Second, there was difference in sides among the three TN groups, and this is maybe a covariant. In the only previous study examining differences in sides, Tsai reported that abnormal brain regions were similar between left and right sides in TN patients (the right side: PFC, precentral gyrus, cerebellar tonsil, and subcortical regions, such as the thalamus, hypothalamus, and nucleus accumbens. the left side: PFC, precentral gyrus, cerebellum, such as tonsil, and so on, and subcortical regions, such as the thalamus, ventral striatum, and putamen). Increasing the sample size may be useful for confirming these results. Third, our grouping of cases according to disease duration was somewhat arbitrary, with a potentially insufficient time between the TN2 and TN3 groups. Finally, although we applied strict correction for multiple comparisons, the sample size was relatively small, and the results may have been affected by methodological error. A meta-analysis reported that most previous studies examining this topic have been based on small sample, typically with approximately 20 subjects. Because of the COVID-19 pandemic, the number of accessible patients is reduced, increasing the difficulty of collecting cases. In further studies, we plan to expand the sample size.

\section{Conclusions}

Most of the variability in previous studies of TN can be explained by classifying TN patients according to disease duration, which allows for more detailed analyses of the mechanisms underlying TN. The results of our study demonstrated that structural alterations occur in specific pain-related brain regions at different stages of the disease and that pain-related matrices involve different stages. The brain automatically filters pain in a bottom-up manner. In the future, further prospective and longitudinal studies could help to elucidate the role of GMV alterations in the pathophysiological processes underlying $\mathrm{TN}$.

Acknowledgements We thank Dr. YX Shen for the assistance in recruiting control subjects, and we thank Benjamin Knight, MSc, from Liwen Bianji, Edanz Editing China (www.liwenbianji.cn/ac), for editing the English text of a draft of this manuscript.

Data availability The datasets which are collected in the current study are available in the picture archive communication system (PACS) repository of the Hebei General Hospital, and datasets analyzed are available from the first author on reasonable request.

Code availability Not applicable.

\section{Declarations}

Conflict of interest The authors declare no competing interests.

Ethics approval All study participants provided written informed consent. The study protocol was approved by Hebei General Hospital Ethics Board.

Consent to participate Informed consent was obtained from all individual participants included in the study.

Consent for publication Patients signed informed consent regarding publishing their data and photographs. 


\section{References}

1. DeSouza DD, Hodaie M, Davis KD (2014) Abnormal trigeminal nerve microstructure and brain white matter in idiopathic trigeminal neuralgia. Pain 155(1):37-44

2. Henderson LA et al (2013) Chronic pain: lost inhibition? J Neurosci 33(17):7574-7582

3. Parise MK, Doring TTA, Tukamoto TM, Vincent G, Gasparetto M, EL, (2014) Cuneus and fusiform cortices thickness is reduced in trigeminal neuralgia. J Headache Pain 15:17

4. Tian T et al (2016) Brain white matter plasticity and functional reorganization underlying the central pathogenesis of trigeminal neuralgia. Sci Rep 6:36030

5. Kuner R, Flor H (2016) Structural plasticity and reorganisation in chronic pain. Nat Rev Neurosci 18(1):20-30

6. Tsai YH et al (2018) Altered structure and functional connection in patients with classical trigeminal neuralgia. Hum Brain Mapp 39(2):609-621

7. May A (2011) Structural brain imaging: a window into chronic pain. Neuroscientist 17(2):209-220

8. Messina R et al (2018) Gray matter volume modifications in migraine: a cross-sectional and longitudinal study. Neurology 91(3):e280-e292

9. Tomasi D, Volkow ND (2019) Association between brain activation and functional connectivity. Cereb Cortex 29(5):1984-1996

10. Tang Y et al (2020) Grey matter volume alterations in trigeminal neuralgia: a systematic review and meta-analysis of voxel-based morphometry studies. Prog Neuropsychopharmacol Biol Psychiatry 98:109821

11. Zhang $C$ et al (2020) Structural and functional brain abnormalities in trigeminal neuralgia: a systematic review. J Oral Facial Pain Headache 34(3):222-235

12. Henssen D et al (2019) Alterations in grey matter density and functional connectivity in trigeminal neuropathic pain and trigeminal neuralgia: a systematic review and meta-analysis. Neuroimage Clin 24:102039

13. Obermann $\mathrm{M}$ et al (2013) Gray matter volume reduction reflects chronic pain in trigeminal neuralgia. Neuroimage 74:352-358

14. Desouza DD et al (2013) Sensorimotor and pain modulation brain abnormalities in trigeminal neuralgia: a paroxysmal, sensory-triggered neuropathic pain. PLoS One 8(6):e66340

15. Zhong J et al (2018) Multivariate pattern classification of brain white matter connectivity predicts classic trigeminal neuralgia. Pain 159(10):2076-2087

16. Gustin SM et al (2011) Different pain, different brain: thalamic anatomy in neuropathic and non-neuropathic chronic pain syndromes. J Neurosci 31(16):5956-5964

17. Zhang $Y$ et al (2018) Dysregulation of pain- and emotion-related networks in trigeminal neuralgia. Front Hum Neurosci 12:107

18. Moayedi M, Desouza D, Erpelding N (2011) Making sense of gray matter abnormalities in chronic orofacial pain-synthesizing divergent findings. J Neurosci 31(35):12396-12397

19. Osborne NR et al (2021) Abnormal subgenual anterior cingulate circuitry is unique to women but not men with chronic pain. Pain 162(1):97-108

20. Reckziegel D et al (2020) Hippocampus shape deformation: a potential diagnostic biomarker for chronic back pain in women. Pain 162(5):1457-1467

21. Rodriguez-Raecke R et al (2009) Brain gray matter decrease in chronic pain is the consequence and not the cause of pain. J Neurosci 29(44):13746-13750

22. Fitzgerald PL, JW; Thakur, PH; Hsiao, SS, (2004) Receptive field properties of the macaque second somatosensory cortex: evidence for multiple functional representations. J Neurosci 24(49):11193-11204

23. Yan CG et al (2016) DPABI: Data Processing \& Analysis for (Resting-State) Brain Imaging. Neuroinformatics 14(3):339-351

24. Eklund A, Nichols TE, Knutsson H (2016) Cluster failure: why fMRI inferences for spatial extent have inflated false-positive rates. Proc Natl Acad Sci U S A 113(28):7900-7905

25. Li M et al (2017) Reduced volume of gray matter in patients with trigeminal neuralgia. Brain Imaging Behav 11(2):486-492

26. Coombes SA, Misra G (2016) Pain and motor processing in the human cerebellum. Pain 157(1):117-127

27. Moulton EA et al (2011) Aversion-related circuitry in the cerebellum: responses to noxious heat and unpleasant images. J Neurosci 31(10):3795-3804

28. Moulton EA et al (2010) The cerebellum and pain: passive integrator or active participator? Brain Res Rev 65(1):14-27

29. Kim J et al (2015) Longitudinal FDG microPET imaging of neuropathic pain: does cerebellar activity correlate with neuropathic pain development in a rat model? Acta Neurochir (Wien) 157(6):1051-1057

30. Gwilym SE et al (2010) Thalamic atrophy associated with painful osteoarthritis of the hip is reversible after arthroplasty: a longitudinal voxel-based morphometric study. Arthritis Rheum 62(10):2930-2940

31. DeSouza DD, Davis KD, Hodaie M (2015) Reversal of insular and microstructural nerve abnormalities following effective surgical treatment for trigeminal neuralgia. Pain 156(6):1112-1123

32. Zhuo M (2008) Cortical excitation and chronic pain. Trends Neurosci 31(4):199-207

33. Hashmi JA et al (2013) Shape shifting pain: chronification of back pain shifts brain representation from nociceptive to emotional circuits. Brain 136(Pt 9):2751-2768

34. Vachon-Presseau E et al (2016) Corticolimbic anatomical characteristics predetermine risk for chronic pain. Brain $139(\mathrm{Pt}$ 7):1958-1970

35. Rutland JW et al (2019) First application of 7-T ultra-high field diffusion tensor imaging to detect altered microstructure of thalamic-somatosensory anatomy in trigeminal neuralgia. J Neurosurg $1-9$

36. Zhuo M (2014) Long-term potentiation in the anterior cingulate cortex and chronic pain. Philos Trans R Soc Lond B Biol Sci 369(1633):20130146

37. Baliki MN et al (2012) Corticostriatal functional connectivity predicts transition to chronic back pain. Nat Neurosci 15(8):1117-1119

38. Wang Y et al (2018) Structural and functional abnormalities of the insular cortex in trigeminal neuralgia: a multimodal magnetic resonance imaging analysis. Pain 159(3):507-514

39. Moon $\mathrm{HC}$ et al (2018) 7 Tesla magnetic resonance imaging of caudal anterior cingulate and posterior cingulate cortex atrophy in patients with trigeminal neuralgia. Magn Reson Imaging 51:144-150

40. Papini MR, Fuchs PN, Torres C (2015) Behavioral neuroscience of psychological pain. Neurosci Biobehav Rev 48:53-69

41. Menon V, Uddin LQ (2010) Saliency, switching, attention and control: a network model of insula function. Brain Struct Funct 214(5-6):655-667

42. Craig A (2009) How do you feel-now? The anterior insula and human awareness. Nat Rev Neurosci 10(1):59-70

43. Apkarian AV et al (2005) Human brain mechanisms of pain perception and regulation in health and disease. Eur $J$ Pain 9(4):463-484 
44. Ourry V et al (2021) Association of quality of life with structural, functional and molecular brain imaging in community-dwelling older adults. Neuroimage 231:117819

45. Rolls ET, Cheng W, Feng J (2020) The orbitofrontal cortex: reward, emotion and depression. Brain Commun 2(2):fcaa196

46. Knudsen EI (2007) Fundamental components of attention. Annu Rev Neurosci 30:57-78

47. Draganski B et al (2006) Decrease of thalamic gray matter following limb amputation. Neuroimage 31(3):951-957

48. Passingham RE, Stephan KE, Kotter R (2002) The anatomical basis of functional localization in the cortex. Nat Rev Neurosci 3(8):606-616

49. Lee JJ et al (2021) A neuroimaging biomarker for sustained experimental and clinical pain. Nat Med 27(1):174-182

50. Pfannmoller J, Lotze M (2019) Review on biomarkers in the resting-state networks of chronic pain patients. Brain Cogn 131:4-9

51. Lotze M, Moseley GL (2015) Theoretical considerations for chronic pain rehabilitation. Phys Ther 95(9):1316-1320

52. Simons LE et al (2012) Fear of pain in the context of intensive pain rehabilitation among children and adolescents with neuropathic pain: associations with treatment response. J Pain 13(12):1151-1161

53. Fasick V et al (2015) The hippocampus and TNF: common links between chronic pain and depression. Neurosci Biobehav Rev 53:139-159

54. Li L et al (2020) Contribution of the P2X4 receptor in rat hippocampus to the comorbidity of chronic pain and depression. ACS Chem Neurosci 11(24):4387-4397

55. Simons LE, Elman I, Borsook D (2014) Psychological processing in chronic pain: a neural systems approach. Neurosci Biobehav Rev 39:61-78

56. Raja SN et al (2020) The revised International Association for the Study of Pain definition of pain: concepts, challenges, and compromises. Pain 161(9):1976-1982

Publisher's note Springer Nature remains neutral with regard to jurisdictional claims in published maps and institutional affiliations. 